\title{
A model of workplace safety incorporating worker interactions and simple interventions
}
J. Thew ${ }^{1}$
T. Bopf ${ }^{2}$
D. G. Mallet ${ }^{3}$

(Received 22 December 2012; revised 30 November 2013)

\begin{abstract}
Although there was substantial research into the occupational health and safety sector over the past forty years, this generally focused on statistical analyses of data related to costs and/or fatalities and injuries. There is a lack of mathematical modelling of the interactions between workers and the resulting safety dynamics of the workplace. There is also little work investigating the potential impact of different safety intervention programs prior to their implementation. In this article, we present a fundamental, differential equation-based model of workplace safety that treats worker safety habits similarly to an infectious disease in an epidemic model. Analytical results for the model, derived via phase plane and stability analysis, are discussed. The model is coupled with a model of a generic safety strategy aimed at minimising unsafe
\end{abstract}

http://journal austms.org.au/ojs/index.php/ANZIAMJ/article/view/6565 gives this article, (c) Austral. Mathematical Soc. 2014. Published January 2, 2014, as part of the Proceedings of the 16th Biennial Computational Techniques and Applications Conference. ISSN 1446-8735. (Print two pages per sheet of paper.) Copies of this article must not be made otherwise available on the internet; instead link directly to this URL for this article. 
work habits, to produce an optimal control problem. The optimal control model is solved using the forward-backward sweep numerical scheme implemented in Matlab.

\section{Contents}

1 Introduction and background

2 Mathematical model

3 Computational implementation

4 Results

\section{Introduction and background}

During the 2009-2010 financial year, out of a total workforce of approximately 12 million Australians, 5.3\% suffered a work-related injury or illness. This resulted in a loss of workplace productivity and cost the Australian government in workplace compensation payouts an estimated $\$ 60.6$ billion or $4.8 \%$ of the gross domestic product of the nation.

Occupational Health and Safety (OHS) was studied relatively intensely for the past four decades with an aim to minimise unsafe work practices and associated costs. Researchers' interests spanned a multitude of different approaches, including observational studies of existing programs [2, 3, 11], developing new approaches to safety improvement, analysis of governmental reforms at both national and state levels $[4,5,10]$ and analysis of data 
regarding OHS costs $[6,7,8]$. However, to the authors' knowledge, there was no attempt to mathematically model interactions between workers and the resulting impact on safety dynamics and costs in the workplace. The apparent shortage of mathematical models presents an opportunity to develop a selection of tools to produce a deeper understanding of the effects of different approaches in minimising OHS costs. Here we present a preliminary model for the dynamics of interacting populations of workers who are classified as either 'safe' or 'unsafe'. The model is proposed as a basis for further investigation regarding occupational health and safety.

The safety habits observed in a workplace depend greatly upon the regulation and maintenance of safety structures provided by management and government legislation. Without regulation, there is potential for unsafe practices to become more common, increasing the risk of injury or illness in the workplace. This results in decreased productivity through a loss of personnel as well as the financial costs associated with sick leave, investigating the incident and training replacement staff. Independently as well as due to government legislation, businesses and industries employ intervention strategies to minimise workplace safety incidents and maximise safety. These methods include OHS legislation [21], on-site health and safety officers [20] and the incorporation of risk management into the agenda of the company [9]. While all of these interventions are in themselves costly, the objective of the company is to seek greater benefit by reducing incident related costs by more than the cost of the intervention itself. An obvious but important pursuit in business practice is to limit the cost of running the company, so mathematical models that are able to describe workplace safety dynamics and investigate the impact of safety programs, a priori, are of great value to industry.

We consider the workforce to be split into four groups: safe and unsafe workers who are currently either on some sort of sick leave or are actively working. The workers interact as part of their daily work activities and are able to alter each others' behaviour, resulting in movement between the different populations. The dynamics are modeled using ordinary differential equations (ODEs) similar to those employed in infectious disease models [13, 16, e.g.]. To incorporate 
safety interventions and to attempt to investigate cost minimisation, we extend the ODE model to an optimal control model.

\section{Mathematical model}

We explore the dynamics of the workforce, in terms of safety classifications, using a modelling strategy that is similar to the SIR models of infectious disease modelling $[13,16]$. Such models were used successfully to model many diseases including the plague $[13,17]$ and measles [19], and modified to model sexually transmitted diseases such as chlamydia [15]. SIR models seek to describe the spread of an infection in a population over time by tracking the populations of susceptible (S), infected (I) and recovered (R) individuals. Infected individuals transmit the infection to the susceptibles through some sort of contact, which is modeled as being proportional to the product of the two populations. Further details on SIR models are found in many sources, but Murray provides an excellent background [16].

The link that we make between SIR models of infectious diseases and the strategy used here to describe worker safety, is to propose that safe behaviour and unsafe behaviour is transmitted in a similar way to the infection in an infectious disease model. That is, safeness is transmitted to unsafe workers at a rate proportional to the product of the safe and unsafe populations. Unsafe behaviour is transmitted in a similar way.

The model involves a system of four ODEs (1)-(4) where $\mathbf{S}(\mathrm{t})$ and $\mathrm{U}(\mathrm{t})$ represent the numbers of workers at time $t$ who undertake their jobs in a mostly safe manner ('safe workers') and unsafe manner ('unsafe workers'), respectively. Similarly, $\mathrm{I}^{\mathrm{S}}(\mathrm{t})$ and $\mathrm{I}^{\mathrm{u}}(\mathrm{t})$ denote the numbers of injured workers who undertake their jobs in a safe or unsafe way, respectively. We assume that new workers are constantly entering the workforce, while workers are fired, injured and die at some rate. Safe and unsafe workers interact, passing on behavioral characteristics related to the safety of their work practices, 
resulting in movement between the two populations. When workers are injured they are no longer actively working; however, after a time they can return to work. Hence, to model the dynamics of the workforce,

$$
\begin{aligned}
S_{t} & =f_{S}+k_{0} S U-k_{1} S U-k_{2} S+k_{4} I^{S}, \\
U_{t} & =f_{U}-k_{0} S U+k_{1} S U-k_{3} U+k_{5} I^{U}, \\
I_{t}^{S} & =\lambda_{S} k_{2} S-k_{4} I^{S}, \\
I_{t}^{u} & =\lambda_{u} k_{3} U-k_{5} I^{u},
\end{aligned}
$$

where $f_{S}$ and $f_{U}$ denote the constant influx of new safe and unsafe workers, $k_{0}$ is the rate at which unsafe workers convert to safe behaviour due to interactions with safe workers, $k_{1}$ is the rate at which safe workers become unsafe due to interactions with unsafe workers, $k_{2}$ is the rate at which safe workers are fired, injured or die and $k_{3}$ is the rate of injury, death or being fired for unsafe workers. The parameters $\lambda_{S}$ and $\lambda_{\mathrm{U}}$ are the fractions of injured safe and unsafe workers, respectively, out of all those who are injured, fired or die, and $k_{4}$ and $k_{5}$ are the rates at which safe and unsafe injured workers, respectively return to their respective uninjured populations. The system is closed by the initial conditions

$$
\mathrm{S}(0)=\mathrm{S}_{0}, \quad \mathrm{U}(0)=\mathrm{U}_{0}, \quad \mathrm{I}^{\mathrm{S}}(0)=\mathrm{I}_{0}^{\mathrm{S}}, \quad \mathrm{I}^{\mathrm{U}}(0)=\mathrm{I}_{0}^{\mathrm{U}} .
$$

The model given by equations (1)-(5) is developed further by introducing a function, $\mathfrak{u}(\mathrm{t})$, which represents an intensity level of a safety intervention program imposed on the workforce at time $t$. The safety intervention function represents the amount of effort (or cost) undertaken to adjust the behaviour of unsafe workers to safer behaviours and is reflected in the adjusted dynamic equations through the term $u(t) U$. We attempt to minimise the number of unsafe workers at some fixed, later time, as well as the costs of the safety program, subject to the dynamics of the workers being governed by the ODE system, and hence we have an optimal control problem. Furthermore, we assume that we have a bounded problem as most companies will have set some maximum quantity of resources available for OHS. Thus we have 
$0 \leqslant \mathfrak{u}(\mathbf{t}) \leqslant \mathfrak{u}_{\max }$, where $\mathfrak{u}_{\max }$ is the maximum amount of resources available for improving safety. The optimal control problem to be solved is

$$
\min _{u} \int_{0}^{t_{\text {end }}} u^{2}(t) d t+u\left(t_{\text {end }}\right), \quad 0 \leqslant u(t) \leqslant u_{\max },
$$

where $t_{\text {end }}$ is the fixed end time for the problem, and subject to equations

$$
\begin{aligned}
S_{t} & =f_{S}+k_{0} S u-k_{1} S U-k_{2} S+k_{4} I^{S}+u(t) u, \\
U_{t} & =f_{u}-k_{0} S U+k_{1} S u-k_{3} U+k_{5} I^{u}-u(t) u, \\
I_{t}^{S} & =\lambda_{S} k_{2} S-k_{4} I^{S}, \\
I_{t}^{u} & =\lambda_{u} k_{3} U-k_{5} I^{u},
\end{aligned}
$$

with initial conditions (5) and free final conditions for all variables.

To solve the optimal control problem, we form the Hamiltonian $\mathrm{H}$ and the Lagrangian L:

$$
\begin{aligned}
\mathrm{H}(\mathrm{t}, \boldsymbol{x}, \mathrm{u}, \sigma) & =\mathrm{u}^{2}+\sigma_{1} \mathrm{~S}_{\mathrm{t}}+\sigma_{2} \mathrm{u}_{\mathrm{t}}+\sigma_{3} \mathrm{I}_{\mathrm{t}}^{\mathrm{S}}+\sigma_{4} \mathrm{I}_{\mathrm{t}}^{\mathrm{u}}, \\
\mathrm{L}(\mathrm{t}, \boldsymbol{x}, \mathrm{u}, \sigma) & =\mathrm{H}(\mathrm{t}, \boldsymbol{x}, \mathrm{u}, \sigma)+\omega_{1}(\mathrm{t}) \mathrm{u}(\mathrm{t})+\omega_{2}(\mathrm{t})\left[\mathrm{u}_{\max }-\mathfrak{u}(\mathrm{t})\right],
\end{aligned}
$$

where $\sigma_{1}, \ldots, \sigma_{4}$ are the adjoint functions, and where the two penalty multipliers $\omega_{1}(t)$ and $\omega_{2}(t)$ satisfy

$$
\begin{aligned}
\omega_{1}(t), \omega_{2}(t) \geqslant 0, & \\
\omega_{1}(t), \omega_{2}(t)=0 & \text { for } 0<u^{*}(t)<u_{\max }, \\
\omega_{1}(t)=0, \quad \omega_{2}(t) \geqslant 0 & \text { for } u(t)=0 \\
\omega_{2}(t)=0, \quad \omega_{1}(t) \geqslant 0 & \text { for } u(t)=u_{\max } .
\end{aligned}
$$

The concavity condition on the Hamiltonian produces $\mathrm{H}_{\mathfrak{u u}}=2>0$, and hence any optimal control will produce a minimum for (6), as required. The 
adjoint equations are then

$$
\begin{aligned}
-\frac{\partial H}{\partial S}=\sigma_{1}^{\prime}(t)= & -\sigma_{1}(t)\left[\left(k_{0}-k_{1}\right) U(t)-k_{2}\right] \\
& -\sigma_{2}(t)\left(k_{1}-k_{0}\right) U(t)-\sigma_{3}(t) \lambda_{S} k_{2}, \\
-\frac{\partial H}{\partial U}=\sigma_{2}^{\prime}(t)= & -\sigma_{1}(t)\left[\left(k_{0}-k_{1}\right) S(t)+u(t)\right]-\sigma_{4}(t) \lambda_{u} k_{3} \\
& -\sigma_{2}(t)\left[\left(k_{1}-k_{0}\right) S(t)-k_{3}-u(t)\right], \\
-\frac{\partial H}{\partial I^{S}}=\sigma_{3}^{\prime}(t)= & k_{4}\left[\sigma_{3}(t)-\sigma_{1}(t)\right], \\
-\frac{\partial H}{\partial I^{\mathrm{u}}}=\sigma_{4}^{\prime}(t)= & k_{5}\left[\sigma_{4}(t)-\sigma_{2}(t)\right] .
\end{aligned}
$$

Equations (17)-(20) are subject to the tranversality conditions

$$
\sigma_{1}\left(t_{\text {end }}\right)=0, \quad \sigma_{2}\left(t_{\text {end }}\right)=1, \quad \sigma_{3}\left(t_{\text {end }}\right)=0, \quad \sigma_{4}\left(t_{\text {end }}\right)=0 .
$$

The optimality condition is

$$
\left.\frac{\partial \mathrm{L}}{\partial \mathrm{u}}\right|_{\mathrm{u}^{*}(\mathrm{t})}=2 \mathrm{u}^{*}(\mathrm{t})+\left[\sigma_{1}(\mathrm{t})-\sigma_{2}(\mathrm{t})\right] \mathrm{u}(\mathrm{t})+w_{1}(\mathrm{t})-w_{2}(\mathrm{t})=0 .
$$

Using the optimality condition, along with equations (13)-(16) we obtain the optimal control

$$
u^{*}(t)=\min \left[u_{\max }, \max \left(\frac{\mathrm{u}(t)\left[\mu_{2}(t)-\mu_{1}(t)\right]}{2}, 0\right)\right] .
$$

When $u=u^{*}$, the system is optimised in the sense of equation (6).

\section{Computational implementation}

Here we present the algorithm (the forward-backward sweep method) used to solve the bounded optimal control problem of Section 2. We adapt the single 
equation scheme outlined by Lenhart [14] to solve the multispecies model proposed here.

Consider the optimal control problem over time interval $\left[t_{0}, t_{1}\right]$, $\max _{\mathfrak{u}} \int_{t_{0}}^{t_{1}} f[t, x(t), \mathfrak{u}(t)] d t \quad$ subject to $\quad x^{\prime}=\mathbf{g}[t, x(t), \mathfrak{u}(t)], \quad x\left(t_{0}\right)=x_{0}$, where $\boldsymbol{x} \in \mathbb{R}^{\mathrm{n}}$ are the state variables, $\mathbf{g}$ is the vector of the right hand sides of the state differential equations, $\mathbf{u} \in \mathbb{R}^{\mathfrak{m}}, \mathrm{n}$ is the number of state equations and $m$ is the number of controls to be implemented on the system.

Introduce matrices $X \in \mathbb{R}^{n \times(N+1)}, \Lambda \in \mathbb{R}^{n \times(N+1)}$ and $U \in \mathbb{R}^{m \times(N+1)}$, where $\mathrm{N}+1$ is the number of time steps to be taken, $\mathrm{X}$ is the approximation to $\mathrm{X}$ at the $\mathrm{N}+1$ time steps, and $\Lambda$ is a matrix of the adjoint equations, such as (17)-(20). Then the following steps solve the optimal control problem.

1. Make an initial guess $\mathbf{U}$ for $\mathbf{u}$ in a given time interval.

2. Using the initial condition $X(1: n, 1)=x\left(t_{0}\right)$ and $U$, solve $X$ forward in time according to its differential equation in the optimality system.

3. Using the transversality condition, such as equation (21), $\Lambda(1: n, N+$ $1)=\boldsymbol{\sigma}\left(t_{1}\right)=0$ (where $\boldsymbol{\sigma}$ are the adjoint functions) and the values for $\mathbf{u}$ and $\mathbf{X}$, solve $\Lambda$ backward in time according to its differential equation, $\boldsymbol{\sigma}$, in the optimality system.

4. Update $\mathbf{u}$ and output the current values as solutions. If values are not sufficiently close, then return to step 3 using the updated $X$ and $\wedge$ values.

5. Check convergence. If values of the control variables in this iteration and the last iteration are negligibly close, then control is optimal.

Computationally, the problem is not expensive to solve. We use standard Matlab ODE solvers to compute results and these are returned usually in 1-2 minutes using an iMac with $3.1 \mathrm{GHz}$ Intel Core i5, 4 Gb $1333 \mathrm{MHz}$ DDR3 memory and Matlab 2012a. 
Table 1: Parameter values used in the solution of the optimal control model obtained from the Australian Bureau of Statistics [1], Safe Work Australia [18] and via calculation and estimation (see main text).

\begin{tabular}{lll} 
parameter & value & source \\
\hline workforce & 10658000 workers & {$[1]$} \\
fatalities & 254 workers per year & {$[18]$} \\
injuries & 153562.5 workers per year & {$[18]$} \\
fired & 600000 workers per year & {$[18]$} \\
$k_{2}+k_{3}$ & 0.06626 per year & Calc. \\
$k_{4}+k_{5}$ & 0.72 per year & Calc. \\
$f_{S}+f_{U}$ & 364020 workers per year & Calc. \\
$\lambda_{S}+\lambda_{U}$ & 0.20371 & Calc.
\end{tabular}

\section{Results}

In this section, the optimal control problem set up in Section 2 is solved using the forward-backward sweep method as discussed in Section 3. First, the parameters of the model are specified in Table 1. Most of the parameters used are drawn from Safe Work Australia reports [18] and the Australian Bureau of Statistics [1].

The parameters that are not taken from the literature are obtained as follows. The rate of workers leaving the workforce is calculated using

$$
k_{2}+k_{3}=\frac{(\text { fatalities }+ \text { injuries }+ \text { fired })}{\text { workforce }} .
$$

To accommodate the returning to work parameters of the model, $k_{4}$ and $k_{5}$, the durable return to work rate is used [12]. The return to work rate states that an average of $72 \%$ of workers returned to work for more than seven months after lodging their claim. As a first approximation we take the recruitment parameters, $f_{S}$ and $f_{U}$, to be the difference in the number of workers leaving the workforce and the number of workers returning to the workforce. The 
two $\lambda$ terms are ratios of the number of workers injured to the number of workers who left the workforce due to injury, death or being fired,

$$
\lambda_{\mathrm{S}}+\lambda_{\mathrm{u}}=\frac{\text { injured }}{\text { injured }+ \text { fired }+ \text { fatalities }} .
$$

For the purposes of this present study, we investigate a workforce over one year, so $t_{\text {end }}=1$, and we impose an arbitrary maximum value for the control of $\mathfrak{u}_{\max }$. The remaining parameters, $\boldsymbol{k}_{0}$ and $\boldsymbol{k}_{1}$, are those which are examined as part of the solution of the model and these are specified in the results.

Initial conditions for the safe and unsafe worker populations are taken as evenly shared quantities of the total workforce in Table 1. Similarly, injured safe and injured unsafe workers are taken as evenly shared quantities from the total number of injured workers in Table 1 . That is, $S(0)=U(0)=5329000$, $\mathrm{I}^{\mathrm{S}}(0)=\mathrm{I}^{\mathrm{U}}(0)=76781$.

There are many parameters in this model which affect the nature of the optimal control and the resulting treatment of the system. These parameters include: (1) the maximum amount of resources that can be expended for safety improvement, $\mathbf{u}_{\max } ;(2)$ the initial numbers of safe and unsafe workers, $\boldsymbol{S}(0)$, $\mathrm{U}(0), \mathrm{I}^{\mathrm{S}}(0), \mathrm{I}^{\mathrm{U}}(0)$; and $(3)$ the affect of varying the values of $\mathrm{k}_{0}$ and $\mathrm{k}_{1}$. In order to develop an overall understanding of the nature of the optimal control, and the effect it has on the workplace safety dynamics, variation to each of these parameters was investigated and will now be discussed.

It is important to understand the general nature of the system before investigating what the effects of varying the parameters might be on the workplace safety system. Figure 1 displays the behaviour of the system when there are no safety programs applied. This figure shows the situation where conversion to unsafe behaviour occurs faster than conversion to safe behaviour. As would be expected, the number of unsafe workers increases while the number of safe workers decreases. The number of injured safe workers decreases sharply while the number of injured unsafe workers also decreases but appears to approach a nonzero equilibrium. Due to the nature of the model, for the reverse situation 


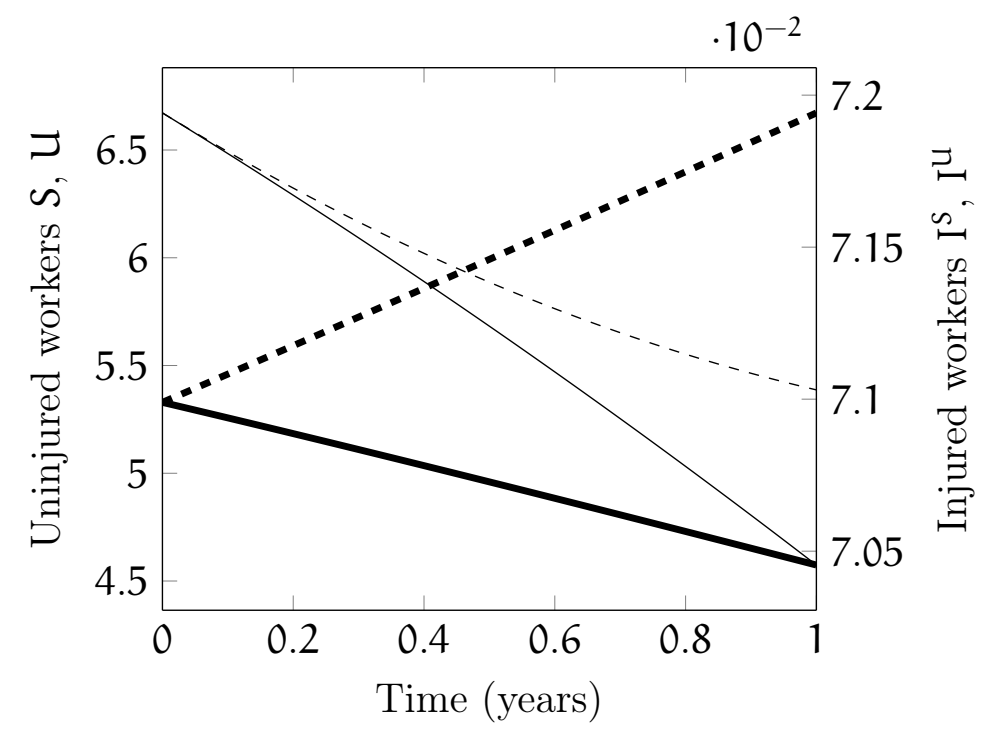

Figure 1: Solution curves $\left(10^{6}\right.$ workers $)$ with no safety interventions. Shown are $\mathrm{S}(\mathrm{t})$ (thick, solid), $\mathrm{U}(\mathrm{t})$ (thick, dash), $\mathrm{I}^{\mathrm{S}}(\mathrm{t})$ (solid), and $\mathrm{I}^{\mathrm{U}}(\mathrm{t})$ (dash) for stronger conversion to unsafe behaviour $\left(k_{1}-k_{0}=0.2\right)$.

where conversion to safe behaviour is faster, the exact opposite situation is observed with safe and unsafe populations switching curves (result not shown).

Now we introduce the safety intervention via the optimal control problem and consider the effect of increasing the amount of resources available to implement the safety program. Figure 2 shows that as the amount of resourcing applied to safety interventions increases, solution behaviour changes markedly. In particular, we note (pleasingly) that the unsafe worker population decreases while the safe worker population increases, with both approaching what appears to be steady levels. Increasing the maximum amount of control beyond that shown in Figure 2 results in a surplus that is not utilised. Solutions quantitatively reproduce those shown in Figure 2. 


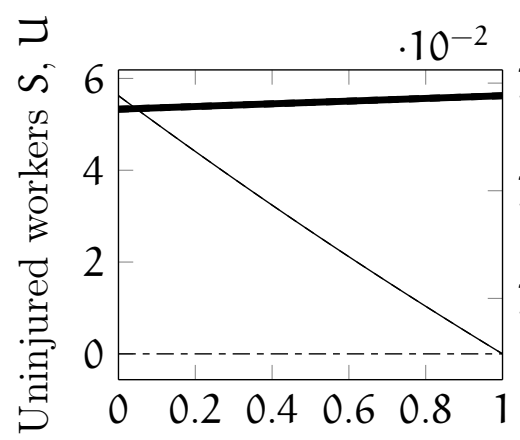

Time (years)

(a) $u_{\max }=0$ (no intervention)

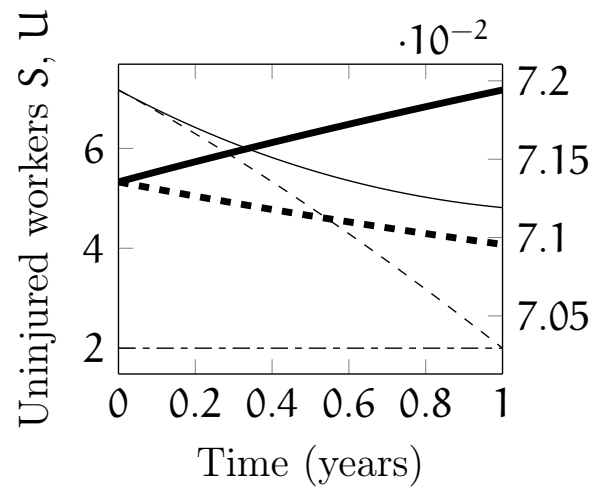

(c) $u_{\max }=2$

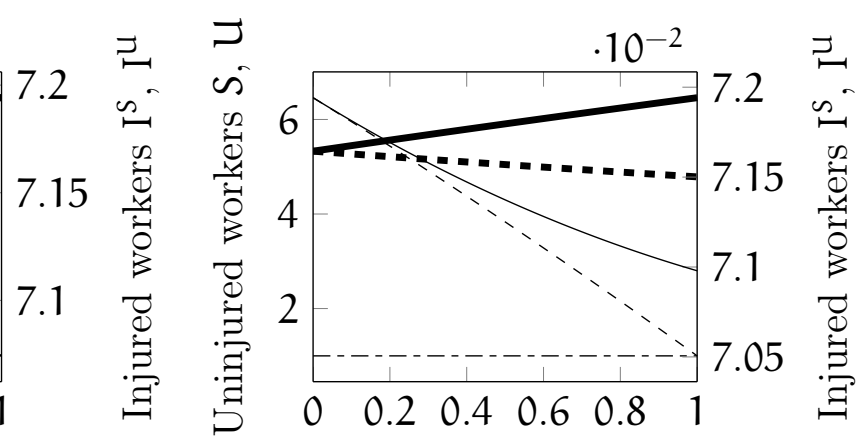

Time (years)

(b) $u_{\max }=1$ 
Next we consider the effects of varying the initial ratio of safe to unsafe workers, which so far has been set at $1: 1$. We investigate two fairly extreme situations of (a) 10\% of the workforce safe and (b) $10 \%$ of the workforce unsafe. For workforces with fewer safe workers than unsafe workers initially, the resourcing of safety interventions is initially high. This leads to fast changes in the workforce, with unsafe workers converting to safer behaviours. The resource requirements of the intervention then decrease as the safe and unsafe populations approach steady levels towards the end of the work year. An example is presented in Figure 3(a) with $10 \%$ of the workforce safe at the start of the project. Resourcing of safety interventions is important here, where unsafe workers outnumber safe workers, as the impact of the program is quite strong. Also, the effects of the safety intervention are maintained in the long term with decreasing levels of resourcing. However, for workforces with initially high levels of safe workers, the impact of the safety programs is weaker. In Figure 3(b) we see the case where initially $90 \%$ of the workforce display safe work habits. Here, there is basically no impact on the worker populations from the safety interventions. It could be argued that any effort or expense resulting from attempting to impose such interventions is wasted.

Finally, we investigate the effects on the population dynamics of varying the interaction rates $k_{0}$ and $k_{1}$. Figures $4-6$ display the results from a number of numerical solutions with varied values for the interaction parameters. For Figures 4 and 5 the safety intervention program coupled with $k_{0}=k_{1}$ or $k_{0}>k_{1}$, respectively, (equal or faster conversion to safe behaviour than unsafe) effectively increases the proportion of workers in the workforce who display safe behaviour. Figure 6 shows that the uninjured unsafe population increases whenever $k_{1}>k_{0}$. Interestingly, despite allowing for a surplus level of resourcing of safety intervention programs, the unsafe worker population continues to increase and there is a resulting decrease in the resourcing of the intervention over time. This result indicates that spending on safety interventions is wasteful if the rate of conversion from safe to unsafe behaviour is too high to be overcome. 


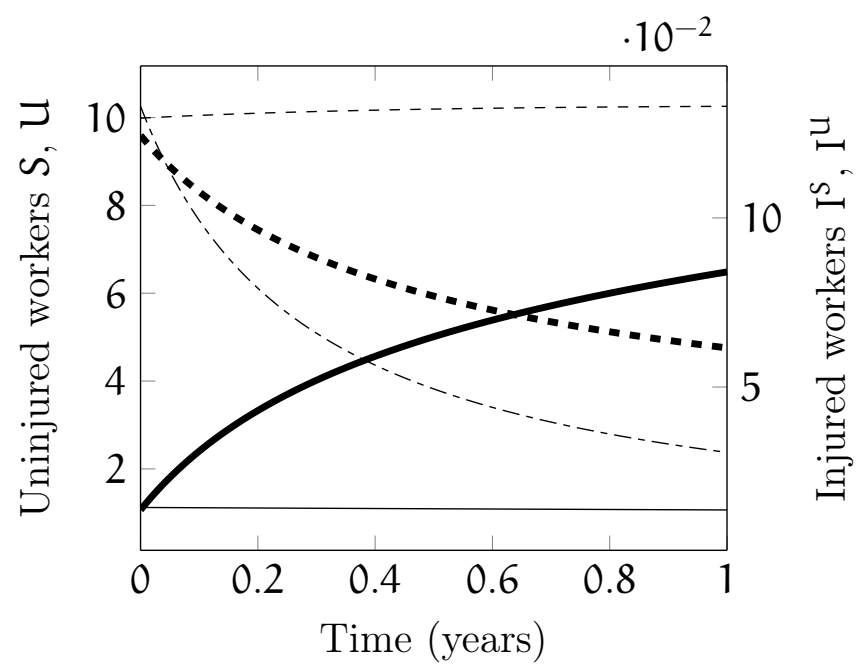

(a) $10 \%$ safe workers

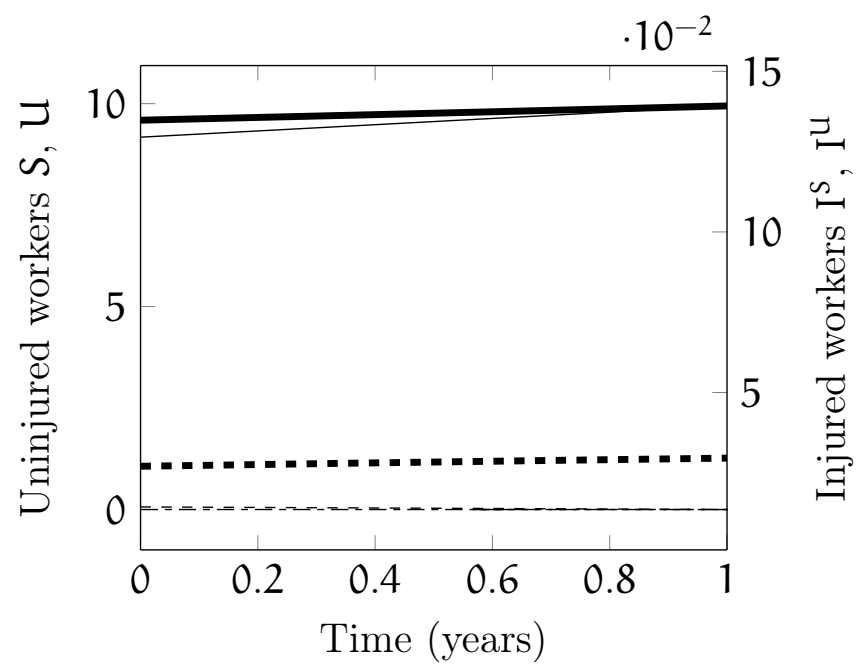

(b) $90 \%$ safe workers

Figure 3: Solution curves $\left(10^{6}\right.$ workers) $\mathrm{S}(\mathrm{t})$ (thick, solid), $\mathrm{U}(\mathrm{t})$ (thick, dash), $\mathrm{I}^{\mathrm{S}}(\mathrm{t})$ (solid), and $\mathrm{I}^{\mathrm{U}}(\mathrm{t})$ (dash) for varying initial ratios of safe: unsafe workers. The optimal control path $\mathfrak{u}(\mathbf{t})$, given by equation (22), is shown as a dotdashed line. 


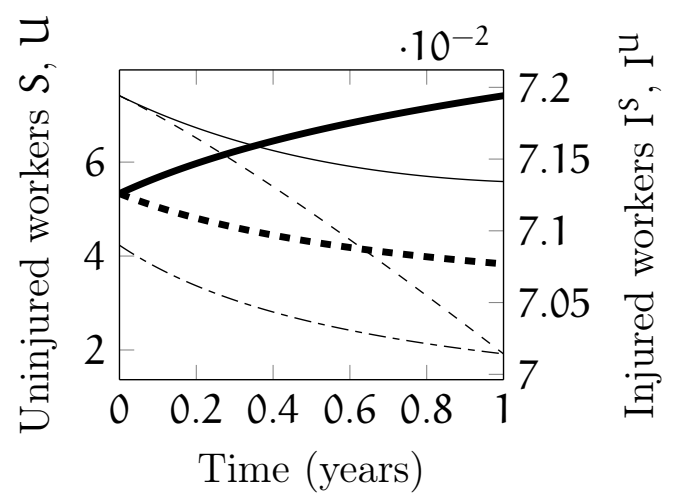

(a) $k_{0}=0.1, k_{1}=0.1$
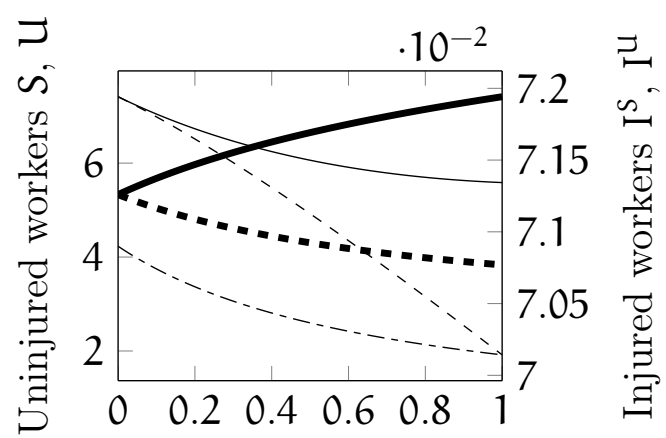

Time (years)

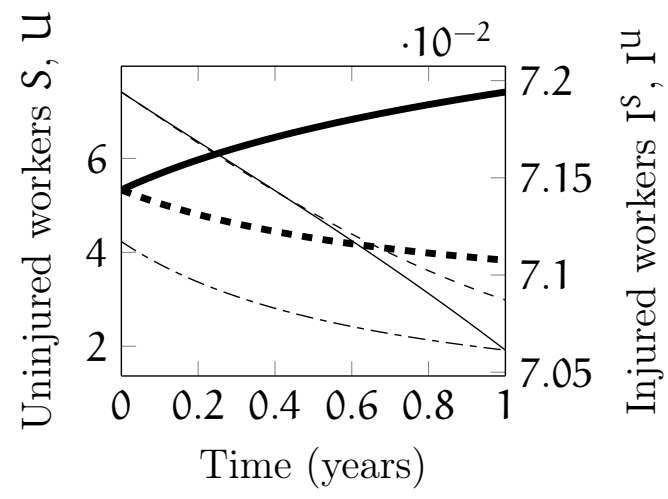

(c) $k_{0}=1, k_{1}=1$

Figure 4: Solution curves ( $10^{6}$ workers) $\mathrm{S}(\mathrm{t})$ (thick, solid), $\mathrm{U}(\mathrm{t})$ (thick, dash), $\mathrm{I}^{\mathrm{S}}(\mathrm{t})$ (solid), and $\mathrm{I}^{\mathrm{U}}(\mathrm{t})$ (dash) for various behaviour conversion rate parameters with $k_{0}=k_{1}$. The optimal control path $\mathfrak{u}(\mathbf{t})$, given by equation (22), is shown as a dot-dashed line. 


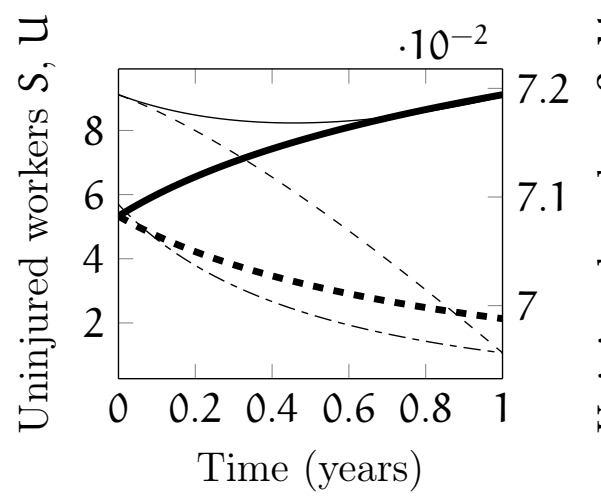

(a) $k_{0}=0.5, k_{1}=0.1$

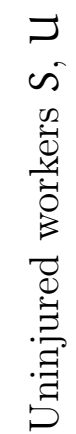

0

$\begin{array}{lllllllll}0 & 0.2 & 0.4 & 0.6 & 0.8 & 1 & \Xi\end{array}$

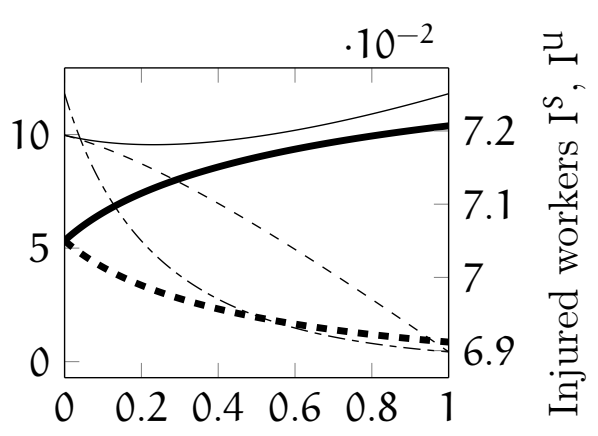

(b) $k_{0}=1, k_{1}=0.1$

Time (years)

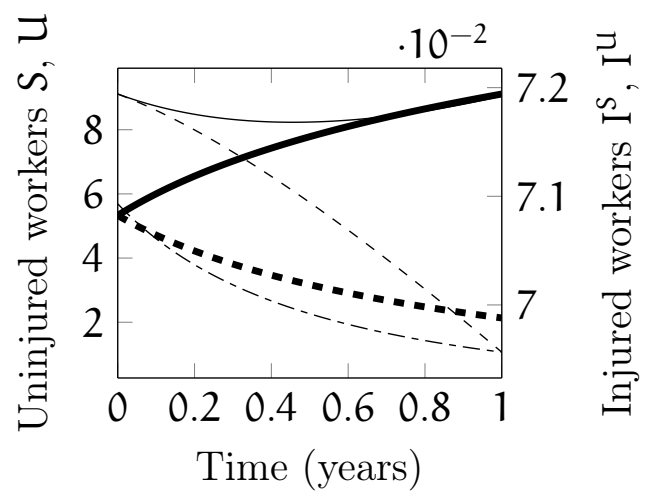

(c) $k_{0}=1, k_{1}=0.5$

Figure 5: Solution curves ( $10^{6}$ workers) $\mathrm{S}(\mathrm{t})$ (thick, solid), $\mathrm{U}(\mathrm{t})$ (thick, dash), $\mathrm{I}^{\mathrm{S}}(\mathrm{t})$ (solid), and $\mathrm{I}^{\mathrm{U}}(\mathrm{t})$ (dash) for various behaviour conversion rate parameters with $k_{0}>k_{1}$. The optimal control path $\mathfrak{u}(\mathbf{t})$, given by equation (22), is shown as a dot-dashed line. 


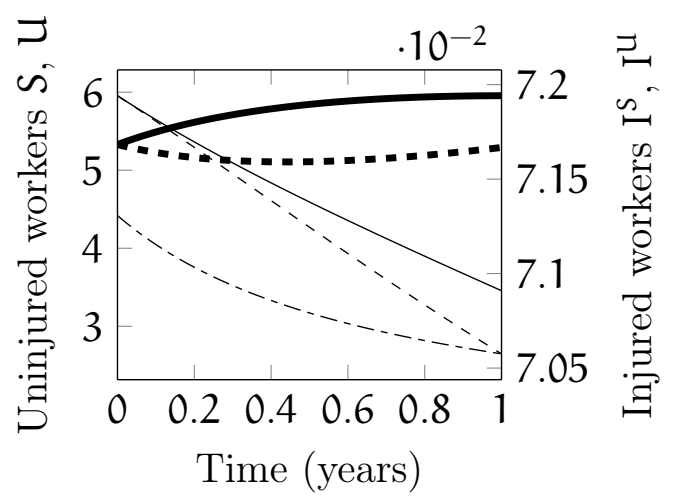

(a) $k_{0}=0.1, k_{1}=0.5$

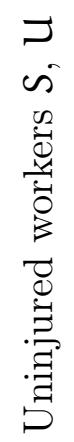

Time (years)

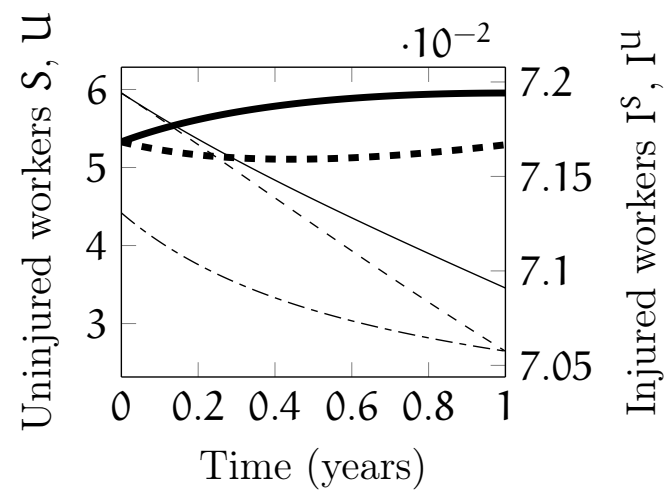

(c) $k_{0}=0.5, k_{1}=1$

Figure 6: Solution curves ( $10^{6}$ workers) $S(t)$ (thick, solid), $U(t)$ (thick, dash), $\mathrm{I}^{\mathrm{S}}(\mathrm{t})$ (solid), and $\mathrm{I}^{\mathrm{U}}(\mathrm{t})$ (dash) for various behaviour conversion rate parameters with $k_{0}<k_{1}$. The optimal control path $\mathfrak{u}(\mathbf{t})$, given by equation (22), is shown as a dot-dashed line. 


\section{Discussion}

We presented a prototype model of the dynamics of populations of workers, classified according to their safety behaviour. The model draws on infectious disease modelling and treats safe and unsafe behaviour similarly to a disease that can be 'caught' from another worker who already displays that behaviour. We also incorporated simple safety intervention programs theoretically via an optimal control problem that seeks to minimise unsafe workers and the costs due to the safety interventions. This model was solved using a multispecies implementation of the forward-backward sweep method for solving optimally controlled systems of ODEs.

Our results focus on investigating the effects of varying the level of resourcing available to safety interventions, the initial ratio of safe to unsafe workers in the workforce, and the safe and unsafe behavioural transmission rates. We found that increasing the amount of resourcing to provide safety interventions is effective in reducing the unsafe worker population toward a stable level that is maintained with reduced levels of resourcing. We also found that for workforces with initially high proportions of safe workers, the impact of safety interventions is quite limited and perhaps not worth the cost of setting up such programs. This could be quite an important finding for industries that are known to have very few unsafe workers. Finally, the investigation of the rates of conversion between safe and unsafe practices indicated that for workforces where the conversion to unsafe behaviour is very high, the safety interventions have little effect on the increasing unsafe worker populations. As such, again, the costs of imposing the type of simple interventions investigated here perhaps outweigh any benefits observed.

A goal of the OHS sector is to reduce the number of safety-related workplace incidents so it is not surprising that substantial effort is directed towards finding the most at-risk industries. In Australia, two such industries are the construction industry and agricultural/logging industries [18]. Additionally, inexperienced workers are also classified as an at risk group for OHS. Future 
research will adapt the modelling work carried out here to investigate such specific industries and worker cohorts.

\section{References}

[1] Australian Bureau of Statistics. Forms of employment. Commonwealth of Australia, 2010. http://www.abs.gov.au/AUSSTATS/abs@.nsf/ DetailsPage/6359. ONovember\%202010?OpenDocument C707

[2] Bahn, S. Power and Influence: Examining the Communication Pathways that Impact on Safety in the Workplace. J. Occup. Health Safety-Aust. N.Z., 25(3):213-222, 2009. C700

[3] Bird, P. Reducing Manual Handling Workers Compensation Claims in a Public Health Facility. J. Occup. Health Safety-Aust. N.Z., 25(6):451-459, 2009. C700

[4] Breslin, P. Improving OHS Standards in the Building and Construction Industry through safe design. J. Occup. Health Safety-Aust. N.Z., 23(4):89-99, 2007. C700

[5] Breslin, P. National Harmonisation: Designers' Duties of Care in the Australian Building and Construction Industry. J. Occup. Health Safety-Aust. N.Z., 25(6):495-504, 2009 . C700

[6] Driscoll, T., Mitchell, T., Mandryk, J., Healey, S., Hendrie, L. and Hull, B. Trends in Work-Related Fatalities in Australia, 1982 to 1992. J. Occup. Health Safety-Aust. N.Z., 18(1):21-33, 2002. C701

[7] Driscoll, T. Fatal Injury of young workers in Australia. J. Occup. Health Safety-Aust. N.Z., 22(2):151-161, 2006. C701

[8] Foley, G., Gale, J. and Gavenlock, L. The Cost of Work-Related Injury and Disease. J. Occup. Health Safety-Aust. N.Z., 11(2):171-194, 1995. C701 
[9] Glendon, I. and Waring, A. Risk management as a framework for occupational health and safety. J. Occup. Health Safety-Aust. N.Z., 13(6):525-532, 1997. C701

[10] Gunningham, N. and Healy, P. Agricultural oHs Policy: Towards Systemic Reform. J. Occup. Health Safety-Aust. N.Z., 20(4):311-318, 2004. C700

[11] Hawkins, A., Eather, J. and Fragar, L. Improving Health and Safety in the Farm Workshop. J. Occup. Health Safety-Aust. N.Z., 24(2):155-160, 2008. C700

[12] Heads of Workers' Compensation Authorities. 2008/09 Australia and New Zealand Return to Work Monitor.

http: //www.hwca.org.au/documents/Australia\%20and\%20New\% 20Zealand\%20Return\%20to\%20Work\%20Monitor\%202008-2009.pdf C707

[13] Kermack, W. O. and McKendrick, A. G. A Contribution to the Mathematical Theory of Epidemics. Proc. R. Soc. Lond. A., 115(772):700-721, 1927. doi:10.1098/rspa.1927.0118 C701, C702

[14] Lenhart, S. and Workman, J. T. Optimal control applied to biological models. Chapman \& Hall CRC Mathematical and Computational Biology Series, 2007. C706

[15] Mallet, D. G., Bagher-Oskouei, M., Farr, A. C., Simpson, D. P. and Sutton, K-J. A mathematical model of Chlamydial infection incorporating movement of Chlamydial particles. B. Math. Biol., 75(11):2257-2270, 2013. doi:10.1007/s11538-013-9891-9 C702

[16] Murray, J. D. Mathematical Biology, I: An Introduction. Springer, 2002. C701, C702

[17] Raggett, G. F. Modelling the Eyam plague. B. I. Math. Appl., 18:221-226, 1982. C702 
[18] Safe Work Australia. The cost of work-related injury and illness for australian employers, workers and the community:2008-2009.

Commonwealth of Australia, 2012. http://www. safeworkaustralia. gov.au/sites/SWA/about/Publications/Documents/660/Cost\% 20of\%20Work-related\%20injury\%20and\%20disease.pdf C707, C716

[19] Shulgin, B., Stone, L. and Agur, Z. Pulse vaccination strategy in the SIR Epidemic Model. B. Math. Biol., 60(6):1123-1148, 1998. doi:10.1006/S0092-8240(98)90005-2 C702

[20] Vanderkruk, R. Workplace health and safety officers: a Queensland success story. J. Occup. Health Safety-Aust. N.Z., 15(6):557-563, 1999. C701

[21] Winder, C. The development of OHS legislation in Australia. J. Occup. Health Safety-Aust. N.Z., 25(4):277-287, 2009. C701

\section{Author addresses}

1. J. Thew, Mathematical Sciences School, Queensland University of Technology, Queensland 4000, Australia.

2. T. Bopf, Mathematical Sciences School, Queensland University of Technology, Queensland 4000, Australia.

3. D. G. Mallet, Mathematical Sciences School, Queensland University of Technology, Queensland 4000, Australia.

mailto:dg. mallet@qut.edu. au 